\title{
Index of Authors, Presenters, Meeting Chairs and Moderators
}

\author{
104th Annual International Conference of the American Society for Horticultural Science
}

16-19 July 2007, Westin Kierland Resort and Spa, Scottsdale, Arizonal

\section{Names are listed in alphabetical order.}

An asterisk (*) indicates a presenting author and these are listed separately.

\section{A}

Abbas, Rana Mazhar, 835, 906

Abdourhamane, Issoufou Kollo, 805

Abe, Kazuyuki, 829, 975

Able, Amanda J., 818

Achor, Diann, 786, 919

Acker, David, 807, 945

Acuna*, Alejandra, 791, 854

Adams, Azure D., 811, 843, 879, 1010

Adkins*, Jeffrey, 788, 929

Agehara, Shinsuke, 811, 842, 879, 1003

Ahmad*, Ishtiaq, 845, 913

Ahmad*, Saeed, 905

Ahmad, Saeed, 835

Ahmad, Hassan, 831, 986

Ahmed*, Waqar, 800, 864

Ahmed, Waqar, 835, 906

Ahn*, Byung Joon, 790, 942

Ajavon*, Amokoe, 831, 989

Ajwa, Husein A., 828, 900

Akagi, Takashi, 829, 976

Al-Mana*, Fahed, 842, 1003

AL-Wasel, Abdelrahman S., 941

Alarcon*, Alejandro, 832

Alarcon, Alejandro, 994

Albano*, Joseph, 937

Albano, Joseph, 789, 806, 839, 840, 872, 937

Albrigo, Gene, 800, 863

Alferez*, Fernando, 871

Alferez, Fernando, 806, 871

Alharbi*, Khadiga, 941

Ali, Muhammad, 835, 906

Alkharouf, Nadim, 822

Allan-Wojtas, Paula, 806, 870

Allsup*, Kelly, 787, 926

Almehdi, Ali, 831, 991

Almenar*, Eva, 816, 884

Alsup, Clydette, 807, 950

Alvarado-Raya*, Horacio E., 786, 920
Alvarez, Hugo, 810, 968

Alvarez, Victor Hugo, 789, 936

Ambruzs, B., 809, 962

Amin, Muhammad, 835, 906

Amissah*, Naalamle, 845, 909

Andersen, Robert, 845, 914

Anderson, Drew, 831, 987

Ando*, Kaori, 873

Ando, Kaori, 806

Andresen, Jeffrey, 789, 842, 940, 1003

Anwar*, Raheel, 835, 906

Anwar, Raheel, 800, 835, 845, 864, 905, 906, 913

Anzai, Hiroyuki, 843, 1007

Appel, Christopher, 944

Aradhya, M., 833

Araki*, Hajime, 952

Araki, Hajime, 808

Arancibia*, Ramon, 790, 850

Araya*, Hintsa, 789, 939

Archbold*, Douglas, 836, 844, 908, 1019

Argo, William, 829, 974

Armstrong, John, 830, 982

Arnold*, Michael, 791

Arnold, Michael, 845, 911

Arnold, Michael A., 828, 898

Arora*, Rajeev, 829, 974

Arora, Rajeev, 819, 822

Arpaia, Mary Lu, 830, 831, 980, 992

Artlip, Timothy, 806, 876

Asagi, Naomi, 808, 952

Asiedu, Samuel K., 788, 928

Astudillo, Paula, 807, 946

Aune, Jeanine, 807, 945

Auras, Rafael, 816, 884

Autenrieth, Robin L., 832, 994

Autio, Wesley, 836, 842, 908, 999

Auwarter, Collin P., 788, 806, 872, 932

Avitia-Garcia, Edilberto, 789, 936
Awan, Faisal Saeed, 835, 906

Ayala*, Santiago, 831, 991

Ayala, Santiago, 787, 843, 924, 1012

Ayeni, Albert, 797, 860

Azarenko*, Anita, 859

Azarenko, Anita, 797, 800, 831, 991

\section{B}

Baameur*, Aziz, 809, 957

Babadoost*, Mohammad, 872

Babadoost, Mohammad, 806

Bachie*, Oli, 852

Bachie, Oli, 790

Bacque*, Lacey Dupre, 952

Bacque, Lacey Dupre, 808

Badertscher*, Kerrie B., 810, 967

Badertscher, Kerrie, 835

Bae, Hae Jin, 811, 879

Bai, J., 818

Bai*, Jinhe, 830, 981

Bai, Jinhe, 817, 833, 835, 842, 902, 1000

Baird, W. Vance, 843, 1007

Baker, David, 944

Bakker*, Cathy, 808, 956

Bakker, Cathy, 808, 957

Balch, Sandra, 832, 900

Baldwin*, E.A., 818

Baldwin*, Elizabeth, 844, 1018

Baldwin*, Patrick, 832, 993

Baldwin, Brian S., 807, 948

Baldwin, Elizabeth, 805, 844, 869, 1018

Bang*, Haejeen, 843, 1013

Barckley, Kristi, 830, 981

Barickman*, Thomas, 831, 986

Barranco, Diego, 827, 895

Barrios-Perez, Agustin, 842, 1000

Barros, Margarita, 805, 870 
Bartens, Julia, 824

Bassett*, Carole, 876

Bassett, Carole, 806, 832, 875, 993

Bassil*, Nahla, 914

Bassil, Nahla, 846, 915

Bassuk, Nina, 824, 845, 909

Bauerle*, William, 790, 810, 832, 849, 965, 966, 993

Bauerle, William, 805, 828, 898

Baugher, Tara Auxt, 842, 1001

Bazan-Tene*, Marcelino, 789

Bazan-Tene, Marcelino, 789, 935, 937

Beam, Donald, 797, 860

Beaman*, Angela R., 788, 931

Beaudry*, Randolph, 811, 882

Beaudry, Randolph, 805, 811, 869, 882

Beddes*, Taun, 832, 996

Beede, Robert, 811, 881

Beeson*, Richard, 800, 862

Beeson, Richard, 800, 828, 861, 862, 898

Behe, Bridget K., 820, 892

Behera*, Tusar Kanti, 846, 915

Bell*, Neil, 950

Bell, Neil, 807

Ben-Yakir, David, 790, 851

Benbrook*, Charles, 825

Benbrook, Charles, 816, 885

Bendik, Elise, 829, 975

Bennett*, Alan, 803

Bennett, Mark, 841, 845, 910, 997

Bergstrom, Gary C., 820, 890

Berkett, Lorranine P., 809, 960

Bernal*, Obdulia Baltazar, 829, 973

Bertolla*, Claudio, 842

Bertolla, Claudio, 1000

Bettmann, Greg, 829, 977

Bewick*, Thomas, 826

Bey, Benjamin, 829, 976

Bhatti, Sarabjit, 844, 1017

Bi*, Guihong, 789, 939

Biasi, Bill, 811, 883

Biernbaum*, John, 838

Biernbaum, John A, 843, 1009

Bilderback, Theodore, 828, 839, 898

Binns, Shannon, 819, 888

Bird, George W., 843, 1009

Bishop*, Garrett, 944

Björkman*, Thomas, 812, 831, 865, 990
Björkman, Thomas, 796, 801

Black*, Brent L., 844, 1022

Black, Brent L., 943

Blackhall, Valeria, 810, 968

Blalock, June, 837

Blanchard, Matthew, 844, 1018

Blount, Ben, 816, 884

Blythe*, Eugene, 789, 937

Blythe, Eugene, 789, 937

Boches*, Peter, 796, 856

Bode, Anne M., 843, 1010

Bogdan*, Sasha, 797, 860

Bomford*, Michael, 808, 831, 954, 988

Bomford, Michael, 843, 1012

Bond, Jason P., 827, 894

Bondarenko, Sveta, 820, 891

Bonds*, Noah, 831, 989

Bonsi, Conrad, 786, 922

Both, A.J., 800, 862

Both, Arend Jan, 806, 875

Botia, P., 790, 849

Boucher*, Alain, 819, 888

Boucher, Alain, 819, 887

Bourget*, Mike, 821

Bowden*, Joseph, 810, 966

Bowden, Joseph, 790, 849

Bowen-O'Connor*, Clare, 789, 790, 829, 941, 942, 977

Boyer*, Cheryl R., 800, 861

Boyer, Cheryl R., 831, 989

Bradeen, James M., 1008

Bradley*, Lucy, 820, 891

Bradshaw, John, 825

Braga, Mike, 831, 991

Branch*, Blake, 809

Branch, Blake, 963

Brand, Mark, 832, 846, 901, 915

Brannon, Laura, 844, 1017

Brevis*, Patricio, 809, 963

Brewbaker*, James, 797, 857

Brewer, Michael J., 843, 1009

Briggs, Jeanne, 786, 920

Brinton, Carrie, 801, 868

Broadley, Martin, 825

Brock, Kathy, 810, 970

Brown*, Patrick, 804

Brown, Patrick, 833, 902

Brown*, Rebecca, 842, 1004

Brumfield*, Robin, 820, 892

Brumfield, Robin, 820, 891
Brutcher*, Lisa, 831, 991

Brutcher, Lisa, 797, 859

Bryant, Heather, 808, 951

Bryla*, David R., 844, 845, 1021, 1022

Bsoul*, Emad, 832, 993

Buchanan*, Gale, 812

Buck*, JohannBrown, Patrick, 833, 902829, 973

Bueren*, Edith Lammerts VanBrown, Patrick, 833, 902813

Bukovac*, Martin J.Brown, Patrick, 833, 902835, 907

Bumgarner*, Natalie, 954

Bumgarner, Natalie, 808

Bunning, Marisa, 809, 958

Burg, Stanley P., 835, 906

Burger*, David, 799

Burnett*, Stephanie, 874

Burnett, Stephanie, 806, 820, 889

Burnett, Arielle, 943

Burns, Jacqueline, 801, 806, 811, 816, $866,871,880$

Burras, C. Lee, 807, 945

Bush*, Edward, 810, 876

Bush, Edward, 810, 876

Busse*, Kendra, 853

Busse, Kendra, 790

Butler, Bryan, 808

Buzby, Karen, 831, 942, 988

Byrne, David, 832, 901

C

Cahn*, Michael D., 828, 900

Cain*, David, 834

Caldeira, Maria Lilia, 845, 912

Caldwell, Claude D., 788, 928

Calhoun, Ron, 831, 990

Cameron, Arthur, 807, 846, 916, 948

Camp, Mary, 808, 953

Campbell, Craig, 805, 806

Campbell, Richard, 841

Campbell-Palmer, Leslie, 806, 870, 871

Cantliffe*, Daniel J., 851

Cantliffe, Daniel J., 786, 788, 790, 835, $842,845,852,907,910,921$, 928, 932, 998

Cantonwine, Emily, 817

Cantor*, Maria, 829, 951, 972 
Cantor, Maria, 807

Cantrell, Charles L., 789, 819, 888, 938

Carbaugh, David, 810, 836, 908, 969

Carey*, Edward, 808, 899

Carey, Edward, 828, 832, 951, 993

Carey, Tamala, 943

Carey*, Ted, 838

Carey, Ted, 816, 885

Carpenter-Boggs, Lynne, 831, 989

Carter*, Steve, 826

Carter, Johnny, 829, 972

Carvalho, Virginia, 829, 974

Casali*, Vicente W.D., 789, 936

Case*, Luke, 832, 995

Case, Luke, 786, 806, 876, 921

Castañeda-Álvarez, Iván, 809

Castellon-Garcia, Luis, 830, 979

Castillo*, Ana Maria, 789, 936

Castillo, Cielo, 789, 833, 905, 941

Castillo*, Nina, 846

Castillo, Nina, 914

Castro, Juan Alberto Osuna, 789, 937

Catanzaro*, Christopher, 812, 844, 1017

Catanzaro, Christopher, 844, 1017

Cathcart, Erin, 796, 807, 854

Cavins, Todd, 844, 1017

Cayanan*, Diane Feliciano, 800, 861

Cerveny*, Christopher, 844, 1016

Chan, Helen, 831, 991

Chandler, Annette, 842, 1004

Chang*, Yao-Chien Alex, 786, 832, 919, 992

Chanon*, Ann, 831, 987

Chao*, C. Thomas, 830, 979

Chaparro, Jose, 842, 999

Chappell*, Matthew, 829, 970

Charudattan*, Raghavan, 817

Chase*, Carlene, 801, 868

Chau, Amanda, 833, 902

Chen*, Chi-Tsun, 830, 980

Chen, Chi-Tsun, 809, 958

Chen*, Chunling, 790, 942

Chen, Chunxian, 833, 904

Chen, Hsiao-Wei, 832, 992

Chen, Jianjun, 846, 917

Chen*, Lianghong, 830, 984

Chen*, Lisong, 836, 909

Chen*, Xun, 951

Chen, Xun, 807, 930

Chen*, Yan, 810, 877
Cheng*, (Max) Zong-Ming, 791

Cheng*, Lailiang, 833, 903, 909

Cheng, Lailiang, 809, 830, 833, 836, 902, 903, 961, 983

Cheol*, Seong Ki, 808, 955

Cheong, Jai Kwon, 836, 909

Cheong, Seoung Ryoung, 806, 872

Cherukuri*, Keerthi, 830, 870, 982

Cherukuri, Keerthi, 805

Chetti, Mahadev, 800, 863

Chiofalo, Francisco, 810, 968

Cho, Hyeon-Mo, 845, 846, 913, 916

Cho, Kwang-Sik, 845, 913

Choi*, Hyun-Sug, 831, 992

Choi*, Jeonghee, 788, 844, 927, 1020

Choi, Cheol, 833, 836, 904, 909

Choi, Ju-Hee, 831, 984

Choi, Kyung Hee, 836, 909

Choi, Seong Yeol, 846, 917

Chong, Calvin, 800, 861

Chozinski, Annie, 797, 831, 859, 991

Chun, Changhoo, 796, 856

Chun, Hee, 786, 923

Chun, Se-Chul, 807, 947

Chung*, Yong Suk, 796, 855

Chung, Jae-Dong, 829, 975

Chung, Kyeong-Ho, 833, 904

Cisneros-Zevallos, Luis, 786, 923

Clancy, Maureen, 833, 903

Clapa, Doina, 829, 972

Clark, Chris, 786, 922

Clark, Christopher, 819, 886

Clark*, David, 803

Clark, David, 808, 953

Clark*, John R., 791, 812, 834

Clark, John R., 809, 812, 961

Clark*, Mary Jane, 831, 987

Clements, Jon, 836, 908

Clivati-McIntyre, Alba, 790, 851

Cloyd, Raymond, 944

Cluj-Napoca, 972

Coffey, Michael D., 843, 1013

Coggeshall*, Mark, 808, 956

Coggeshall, Mark, 956

Coker, Christine, 789, 938

Colavita, Graciela, 810, 968

Cole*, Janet, 791

Cole, Janet, 785, 841, 846

Collier-Christian, Lucy, 832, 994

Collin, Sue, 830, 980

Collins*, Claudia, 790, 853
Collins, Claudia, 807, 944

Collins*, Pamela, 810, 967

Compton*, Michael, 827, 841, 873, 874, 893

Compton, Michael, 806, 873

Connell*, Joseph, 798

Conner*, Patrick, 788, 798, 809, 834, 930, 963

Conner, Patrick, 797, 799

Contreras*, Samuel, 841, 845, 910, 997

Cook*, Emily K., 953

Cook, Emily K., 808

Copeland, Brian, 844, 1017

Cortell*, Jessica, 858

Cortell, Jessica, 797

Cortés-Sánchez, Adriana Aurora, 843, 1008

Cotton, Corrie, 789, 938

Coulter*, Sarah K., 818

Counts, James W., 790, 851

Cousins, Matthew, 786, 920

Cowan*, Janice, 790, 853

Cowgill*, Winfred, 836, 842, 908, 999

Crabtree*, Sheri, 811, 881

Crabtree, Sheri, 789, 880, 939

Craig, Richard, 809, 963

Cramer*, Christopher, 843, 1011

Cramer, Christopher, 836, 844, 1015

Cregg, Bert, 789, 800, 810, 861, 878, 940, 965

Criley*, Richard A., 844, 1015

Crosby*, Kevin, 843, 1013

Crosby, Kevin, 809, 843, 961, 962, 963, 1006

Crouse, K. Kieth, 831, 989

Cruz-Castillo, Juan Guillermo, 843, 1008

Cruz-Huerta*, Nicacio, 786, 920

Cuevas*, Hugo E., 843, 1013

Cummins, John, 786, 921

Cuthbert*, Cheryl, 841, 997

D

Dai*, Wenhao, 789, 833, 905, 941

Dami*, Imed, 809, 961

Dami, Imed E., 797, 858

Damicone, John P., 843, 1010

Dandekar*, Deepak, 816, 885 
Dandorf*, Alan, 830, 984

Dane*, Fenny, 827, 895

Dane, Fenny, 827, 845, 894, 911

Daniel*, Kyle, 876

Daniel, Kyle, 806

Daniels, Lee, 842, 1005

Dar*, William, 805

Darnell, Rebecca, 786, 830, 920, 984

Daugovish, Oleg, 808, 953

Davenport*, Joan, 823, 831

Davenport, Joan, 828, 896

Davenport*, Thomas L., 835, 906

Davenport, Thomas L., 905

Davies*, Fred T., 832, 994

Davies, Fred, 785, 786, 833, 902, 923

Davis*, Angela, 802, 843, 1012

Davis, Angela, 788, 802, 827, 896, 927

Davis*, Donald R., 825

Davis, Ed, 827, 895

Davis*, Karen, 787, 925

Davis, Lei Lani, 801, 868

Davis, Mark, 808, 953

Davis, Thomas, 833, 846, 903, 915

Day, Susan, 824, 828, 898

Day, Toby, 829, 977

Dee, M., 788, 933

DeEll*, Jennifer, 830, 980

DeEll, Jennifer, 824, 828

Deewatthanawong*, Rujira, 830, 982

DeJong, Theodore, 811, 881

Delannay, Isabelle, 796, 856

Delate*, Kathleen, 801, 867

Delate, Kathleen, 801, 867

del Pozo, Alejandro, 800, 864

Demchak*, Kathleen, 830, 838, 983

Demchak, Kathleen, 844, 1021

DeMoranville*, Carolyn, 828, 896

Deng*, Zhanao, 829, 971

Dennis*, Jennifer, 842, 1002

Dennis, Jennifer, 796

Denny*, Geoffrey, 845, 911

Detweiler, Amy Jo, 790, 853

Devine, Carolyn, 820, 892

DeVos, Neal, 835

Deyton, Dennis, 786, 807, 921, 946

De la Rosa, Raúl, 827, 831

Dhanaraj1, Anik L., 822

Dhekney, Sadanand, 797, 858

Dhir*, Ritu, 948

Dhir, Ritu, 807, 846, 916
Dias, José, 829, 974

Diaz, Juan Carlos, 826

Diaz-Perez, Juan, 797

Dickinson*, Sarah, 842, 1005

Dietrich*, Katherine, 944

Dietrich, Katherine, 786, 923

Dighton, John, 800, 862

Dilley*, Craig, 809, 960

Dinsmore*, Jonathan, 954

Dinsmore, Jonathan, 808

Dixon, Michael, 800, 861

Dobres*, Michael, 804

Dobres, Michael, 821

Dodson*, Hallie, 811,878

Dole, John, 820, 889, 890

Domínguez-Camarena, Ernesto Arturo, 789, 935

Doncaster, Kristen, 806, 871

Dong, Weibo, 817

Donoso*, Teresa Salame, 946

Donoso, Teresa Salame, 807

Dossett*, Michael, 828, 897

Doucette, Craig, 806, 870

Dougher*, Tracy, 829, 836, 977

Dougher, Tracy, 836

Douglass*, Cameron, 786

Douglass, Cameron, 921

Dove, Joe, 824

Downer*, A. James, 810, 830, 878, 978

Downer, A. James, 808, 810, 830, 877, 953

Dozier, William, 805, 830, 870, 980, 982

Du, Lanying, 930

Duclos*, Denise, 801, 865

Dumitras, Adelina, 807, 951

Dunn*, Bruce, 832, 900

Durand*, Karla Deza, 788

Durand, Karla Deza, 928

Durham*, Richard, 810, 966

Durst, Robert, 843, 1008

Dutt, Manjul, 797, 858

\section{E}

Ebel, , Robert, 805, 830, 870, 979, 982

Ebeler, Susan, 811, 882

Ebelhar, M. Wayne, 789, 938

Eddy, Marlys, 809, 960
Edney, Sharon, 806, 875

Edwards*, Diane, 786, 922

Egel, Daniel, 788, 932

Egilla*, Jonathan N., 789, 936

Egnin*, Marceline, 829, 976

Eguchi, Sai, 829, 976

Ehlenfeldt*, Mark, 844, 1021

Ehlenfeldt, Mark, 823, 830, 982

Ehret, David, 786, 922

Elfving, Don, 810, 969

Eljak, Intsar, 789, 938

Ellis, Amy, 789, 940

Ellis, Dave, 809, 963

Elsner*, Karen, 788, 930

Enciso, Juan, 809, 964

Etlinger, Melissa, 816, 885

Etxeberria*, Ed, 786, 919

Eum*, Hyang Lan, 787

Eum, Hyang Lan, 927

Evans, Michael, 829, 973

Evans, Richard, 789, 940

Evans, William, 789, 834, 835, 938

Eversole*, Kellye, 821

F

Faber, Ben, 789, 940

Fadamiro, Henry, 830, 980

Fain*, Glenn B., 831, 989

Fain, Glenn B., 800, 861

Fallahi, Bahar, 907

Fallahi*, Esmaeil, 836, 907

Fan*, Lihua, 870

Fan, Lihua, 806

Fargo*, Erik, 788

Fargo, Erik, 929

Farias-Larios, Javier, 789, 935, 937

Farnham*, Mark, 827, 895

Farnham, Mark, 785, 837

Farrell, Jr., Robert, 806, 876

Faust, James, 820, 889

Felicetti, David, 836, 909

Felker*, Peter, 827

Fellman*, John K., 840

Fennimore, Steven, 828, 900

Fernandez, R. Tom, 800, 861

Fernandez, Tom, 789, 940

Ferriss*, Terry, 827, 893

Ferriss, Terry, 785 
Fidelibus*, Matthew, 811, 880

Finlayson, Scott, 833, 902

Finn, Chad, 828, 897

Fisher, Luci, 844, 1018

Fisher, Paul, 829, 974

Fisk, Connie, 796

Fitzgerald, Jay, 831, 987

Fitzpatrick*, George, 827, 893

Fitzpatrick, George, 785

Fixen*, Paul, 812

Fjeld, Kent, 830, 980

Flanagan, P.C., 788, 933

Flore, Jim, 800, 864

Follett, Peter, 830, 982

Folta*, Kevin, 822, 833, 903

Fonseca*, Jorge, 790, 816, 831, 854, 884,984

Fonseca, Jorge, 944

Fonseca-Gongora, Roberto, 830, 979

Forsline, Philip, 805, 827, 869, 895

Foshee, W.G., 808, 952

Francis*, David, 790, 851

Francisco*, Radillo-Juarez, 788, 958

Franco-Mora*, Omar, 843, 1008

Franklin, Emma, 854

Franklin, Jason, 819, 886

Franks*, Cleve, 817

Frantz*, Jonathan, 844, 1018

Frantz, Jonathan, 806, 820, 826, 846, 874, 890, 918

Fraunhofer, J.A. von, 990

Frazier*, Mary Jo, 787, 926

Freed, Trish, 810, 967

Friend*, Alexander, 799

Frietze*, Victoria, 842, 1002

Frisby, James W., 844, 943, 1022

Fritz*, Vince, 843, 1010

Fritz, Vincent A., 843, 1011

Fu, Jianmin, 930

Fu, Junqiu, 845, 912

Fuchigami, Leslie, 789, 939

Fuchs*, Marc, 804

Fulcher*, Amy, 789, 940

\section{G}

Gal, Elazar, 790, 851

Gallagher, Thomas V., 800, 861

Galli*, Federica, 844, 1019
Gan, Jay, 789, 820, 845, 892, 911, 940

Gann, Ashley, 809, 964

Gao, Hui, 829, 976

Garcia*, M. Elena, 809, 960

Garcia-Sanchez, Paco, 790, 849

Garrison, Stephen, 843, 1009

Garriz*, Patricia, 810, 968

Gasic*, Ksenija, 843, 1007

Gasic, Ksenija, 829, 832, 975, 993

Gaumond, Ruth, 791

Gebhardt, Susan, 825

Gee, Wai, 831, 991

Geier, Brian, 831, 988

Geneve*, Robert, 840

Geneve, Robert, 789, 940

Geng*, Fang, 930

Getter, Kristin, 842, 1003

Giacomelli*, Gene, 837

Gige, Alexander Joel G., 805, 869

Gilbert, Kristine, 9789, 40

Gilbertson, Robert L., 805

Gilliam, Charles H., 800, 831, 861, 989

Gilman, Edward, 809, 965

Gilreath, Phyllis, 811, 879

Giovanni, Maria, 809, 957

Giovanni, Piccinni, 842, 1002

Girennavar*, Basavaraj, 841, 997

Gladon, Richard J., 788, 931

Glenn, David Michael, 801, 865

Gliessman, Steven, 808, 952

Gmitter*, Frederick, 814, 904

Gmitter*, Frederick, 833

Gmitter, Julie, 833, 904

Goebel*, Jessica, 944

Goktepe, Fahrettin, 829, 971

Goldman, Irwin, 790

Goldner*, William, 826

Goldway, Martin, 833, 903

Gonsalves*, Dennis, 803

Gonzales*, Sandra, 829, 842, 977, 1005

Gonzalez, Pedro, 786, 919

González-González*, Juan Manuel, 830, 842, 979, 1000

González-González, Juan Manuel, 788, 809, 843, 931, 958, 1008, 1011

Goo*, Dae Hoe, 820, 890

Goo, Dae Hoe, 846, 917

Gooch*, Nicholas, 810, 965

Goodner, Kevin, 805, 844, 869, 1018

Goodson, Tony, 844, 1020
Gordon, Jeffrey S., 805

Gossen, Bruce, 788, 933

Govindasamy, Ramu, 797, 860

Grace, John, 837

Gradziel, Thomas, 798

Graetz, Donald, 832, 996

Graham, Thomas, 800, 861

Gray*, Dennis, 858

Gray, Dennis, 797

Greco, Steve, 845, 911

Greene*, Duane, 830, 836, 842, 908, 984,1001

Greene, Duane, 835, 907

Gresham, Charles, 786, 920

Griesbach, Robert, 829, 843, 971, 1006

Grieve, Catherine, 842, 1002

Griffin*, Jason, 788, 931

Griffin, Jason, 823, 828

Grijalva-Contreras*, Raul Leonel, 786, 787, 843, 924, 1009

Grijalva-Contreras, Raul Leonel, 808, 809, 955, 960

Gross, Kenneth, 844, 1014

Grosser*, Jude, 833, 904

Grossman*, Yaffa, 799

Grube*, Rebecca, 813

Grumet, Rebecca, 806, 873

$\mathrm{Gu}^{*}$, Mengmeng, 787

Gu, Mengmeng, 787, 925, 926

$\mathrm{Gu}^{*}$, Sanjun, 809, 960

Guaqueta, Nelson, 835, 906

Gucci*, Riccardo, 790, 842, 850, 1000

Guckenberger*, Julie, 843, 1006

Guerrero, Cosme, 787, 831, 924, 991

Guerrero, Jose, 843, 1012

Guertal*, Elizabeth, 794

Guinan, Patrick, 795

Gulia*, Surinder, 829, 972

Gunter*, Christopher, 788, 932

Guo, Baozhu, 817

Guo, Ling, 790, 829, 942, 978

Guy, Charles, 830, 982

Guzman-Gonzalez*, Salvador, 830, 842, 843, 1000, 1008

Gwinn, K.D.,788, 933

H

Haffner, Ted, 824 
Hammond, John, 825

Hampson*, Cheryl, 831, 988

Han*, Jeung-Sul, 833, 904

Han, Jeung-Sul, 829, 975

Han, Kyoung Suk, 806, 872

Han*, Yuepeng, 829, 975

Hancock, James, 809, 963

Handley*, David, 830, 983

Handley, David T., 808, 951

Handwerker*, T.S., 831, 989

Hanscom, Janice, 844, 1016

Hanson*, Eric, 828, 898

Hanson, Eric, 796, 811

Hara, Hiromichi, 843, 1007

Harbage, James, 788, 929

Harbaugh, Brent, 829, 971

Harbut*, Rebecca, 828, 830, 897, 983

Hardy*, Whitney, 943

Harkess*, Richard L., 846, 916

Harkess, Richard, 785, 791, 807, 948

Harnly, James, 825

Harper, Carol, 788, 928

Harris*, J. Roger, 824, 829, 978

Harris, J. Roger, 807, 828, 842, 898, 950,1005

Harris, Robert, 819, 888

Harsh, R. Matt, 842, 1001

Harte, Bruce, 816, 884

Hartz*, Timothy K., 794

Hashem, Fawzy, 789, 938

Hashimoto, Fumio, 829, 971

Hassan, Saad, 846, 918

Hassell*, Richard, 802

Hatterman-Valenti*, Harlene M. 788, $872,887,932$

Hatterman-Valenti, Harlene, 806, 819, 871

Haub, Mark, 807, 946

Hausbeck, Mary K., 790, 850

Haver, Darren, 820, 845, 892, 911

Havercamp, Michael, 810, 967

Hayes*, Ryan, 828, 896

Hayes, Ryan, 799, 813

Haynes*, Cynthia, 836

Haynes, Cynthia, 810, 820, 891, 966

Haytowitz, David, 825

He*, Chuanjiu, 832

He, Chuanjiu, 786, 833, 902, 923, 994

He, Fang, 819, 888

He, Zhongqi, 806, 874
Heath, Robert, 831, 992

Heinz, Kevin, 833, 902

Hellier, Barbara, 826, 828

Hendrickson*, Christopher, 842, 1004

Henk, Adam, 827, 895

Hennigan, Kelly, 841

Heo*, Seong, 846, 916

Hernandez-Maruri, Jose Alfredo, 789, 936

Hesterberg, Dean, 839

Hilaire, Rolston St., 941, 942, 977

Hill*, Jamison, 831, 989

Hill, Jill, 786, 922

Hill, Walter, 786, 922

Hirata, Toshiyuki, 808, 952

Hird*, Abby, 827, 894

Hirschi*, Kendal, 792

Hirschi, Kendal D., 833, 904

Hochmuth, George, 801, 868, 879

Hochmuth, Robert, 811, 868, 879

Hodel* $^{*}$, Donald R., 810, 877

Hodel, Donald R., 810, 830, 878, 978

Hodges*, D. Mark, 870

Hodges, D. Mark, 805, 844, 869

Hofmann, Christa, 842, 1002

Hokanson*, Stan C., 831, 986

Hokanson, Stan C., 846, 915

Hoke, Sara, 788, 932

Holbrook*, C. Corley, 817

Holden*, Joanne, 825

Holder, Charles, 829, 842, 977, 1005

Holle, Betsy Von, 807, 950

Hollingsworth, Amelia, 855

Hollingworth, Robert, 810, 878

Holloway*, Patricia, 844, 1016

Holman, G., 809, 962

Holtz, Bren, 808, 831, 957, 991

Hong*, Jeong, 845, 910

Hong*, Jiyoung, 842, 998

Hong, Chuanxue, 828, 898

Hong, Gyung-Hy, 845, 913

Hoover*, Emily, 947

Hoover, Emily, 807, 842, 999

Hopkins, Donald, 797, 858

Horacio E. Alvarado-Raya*, 920

Horneck*, Donald, 812

Howlett*, Teri, 810, 877

Hoy, Mary, 786, 819, 886, 922

Hoying, Stephen, 845, 914

Hoyt*, Greg, 843, 1012
$\mathrm{Hu}^{*}$, Dongyan, 845, 912

$\mathrm{Hu}$, Jinguo, 809, 963

Hu, Yanli, 829, 978

Hua, Sui Sheng T., 831, 991

Huan, Jin, 809, 959

Huang*, Lingyu, 843, 1012

Huber*, Donald, 788, 927

Huber, Don, 811, 883

Huber, Gordon, 809, 959

Hudson, Peter M., 831, 989

Huffman*, Wallace, 803

Hughes*, Harrison, 841, 996

Hughes, Harrison, 788, 930

Huh*, Eun Joo, 846, 917

Hummer*, Kim E., 843, 1008

Hummer, Kim, 846, 915

Humpal*, Donald, 816, 884

Humpal, Don, 816, 884

Hurr*, Brandon, 811, 883

Hutchinson*, Chad M., 794

Hutchinson, Chad, 790, 811, 852, 878

Hutton*, Mark, 830, 951, 983

Hutton, Mark G., 808

Hwang*, Byung-Ho, 844, 1014

\section{I}

Igasaki, Tomohiro, 829, 975

Ikegami, Ayako, 829, 976

Ikemura, Yoshiaki, 814

Incer, M. Regina, 832, 995

Ingham, Russ, 831, 991

Ingram*, David M., 831, 989

Ingram, Dewayne, 805

Inoue*, Eiichi, 843, 1006

Iqbal, Zahid, 800, 864

Itai, Akihiro, 811, 880

Iván, Castañeda-Álvarez, 958

Iwanami, Hiroshi, 829, 975

Izumi*, Hidemi, 818

J

Jabbar, Abdu, , 835, 906

Jack, Allison, 801, 867

Jackson*, Brian, 832, 996

Jaeckel, Bradley, 819, 887

Jahn*, Molly, 805, 813 
James, Bryson, 791

Jarret, Robert L., 841

Jauron*, Richard, 810, 966

Jayaprakasha*, G.K., 816, 885

Jayaprakasha, G.K, 800, 819, 831, 863, 887,985

Jeliazkova*, Ekaterina, 810, 967

Jenderek*, M.M., 809, 962

Jenderek, M.M., 817

Jenderek, Maria, 819

Jenkins, Tracie, 829, 970

Jenks, Mathew, 829, 975

Jeong*, Jin-Cheol, 844, 1020

Jeong, Mooncheol, 844, 1020

Jeong, Sang-Bouk, 845, 913

Jeong, Se-Woon, 829, 975

Jesudhasan, Palmy R., 829, 976

Jett*, Lewis, 786, 924

Jiang*, Zhongchun, 812

Jifon*, John, 788, 809, 934, 964

Jifon, John, 842, 843, 1002, 1013

Jiménez*, Alfredo López, 786

Jiménez, Alfredo López, 920

Jin*, Xiaoling, 929

Jin, Xiaoling, 788, 931

John-Karuppiah*, Karthik-Joseph, 801, 866

Johnson*, Holly, 801, 865

Jolliffe, Peter, 786, 922

Jones, Joy, 790, 853

Jones*, Stephen, 814

Jourdan, Pablo S., 831, 987

Jovicich*, Elio, 790

Jovicich, Elio, 851

Joyce, Daryl, 842, 1004

Jull*, Laura, 810, 877

Jung*, Seok-Kyu, 811, 882

Justen*, Veronica L., 843, 1011

Justen, Veronica L., 843, 1010

Juvera*, Jose, 924

Juvera, Jose, 787, 831, 843, 991, 1012

\section{K}

Kabelka*, Eileen, 1014

Kabelka, Eileen, 843, 1014

Kahane*, Remi, 804

Kahn*, Brian, 836, 843, 1010

Kai, Takanori, 831, 986
Kalberer*, Scott, 829, 974

Kallsen*, Craig, 798, 808, 957

Kallsen, Craig, 808, 957

Kamal, Neel, 843, 1011

Kan*, Elena Lon, 788

Kan, Elena Lon, 928

Kane*, Brian, 829, 978

Kang*, Jong-Goo, 820, 889

Kang*, Sam-Sog, 845, 913

Kang, Sam Sog, 846, 916

Kang*, Won Hee, 842, 844, 998, 1014

Kang, Yejeong, 844, 1020

Karcher*, Doug, 815

Karlsson*, Meriam, 787, 924

Katz, Sidney, 819, 888

Katzir, Nurit, 843, 1012

Kaufman, Diane, 845, 1022

Kays, Stanley, 807, 947

Kean, Deborah, 813

Keathley, Craig, 788, 932

Keller, Markus, 823

Keller, William, 789, 939

Kelley, Rowena Y, 789, 941

Kelly, Jack J., 789, 940

Kemble, J.M., 808, 952

Kenny, Tim, 807, 947

Khan*, Muhammad Mumutaz, 835, 906

Khan, Muhammad Mumutaz, 800, 835, 864,905

Kibet*, Leonard, 789, 938

Kidou, Shin-ichiro, 829, 975

Kikkert*, Julie, 843, 1010

Killough, Cynthia, 789, 790, 829, 941, 942977

Kim*, Chang Kil, 829, 975

Kim*, Hark-Joo, 786, 923

Kim*, Hye-Ji, 787, 846, 917, 926

Kim*, Hyun-Jin, 831, 984

Kim*, Jin Hee, 831, 985

Kim*, Jongkee, 844, 1014

Kim*, Wol-Soo, 810, 970

Kim, Byeongsam, 788, 927

Kim, Chang-Kil, 833, 904

Kim, Chun Hwan, 808, 955

Kim, Chun Hwon, 830, 981

Kim, Dongman, 844, 1020

Kim, Hyun-Jin, 816, 884, 944

Kim, Hyun Ran, 846, 916

Kim, Ju Sung, 844, 1020

Kim, Ki-Taek, 796, 856
Kim, Ki Deog, 844, 1020

Kim, Myung-Su, 845, 913

Kim, Seong Cheol, 830, 981

Kim, Seon Ki, 790, 942

Kim, Yoon-Kyeong, 845, 913

Kim, Zhoo-Hyeon, 809, 961, 962

Kimball, Bruce A., 786, 787

Kime, Lynn, 831, 992

King*, Stephen, 802

King, Stephen, 802, 842, 843, 1001 , 1012, 1013

Kitajima, Akira, 829, 976

Kitto, Sherry, 788, 929

Kjelgren*, Roger, 842, 845, 1004

Kjelgren, Roger, 845, 910

Klaine, Stephen J., 842, 1003

Klassen, Waldemar, 844, 1014

Klee, Harry, 835, 907

Kleinhenz*, Matthew, 797, 801, 851, 859,868

Kleinhenz, Matthew, 790, 797, 846, 860

Klett*, James, 832, 995

Klett, James, 810, 877

Kline*, Welsey, 843, 1009

Kline, Wesley, 815, 847

Kling, Gary, 787, 926

Klingeman*, W.E., 788, 933

Klooster*, Wendy, 800, 861

Knerr, Larry, 836

Knewtson*, Sharon, 828, 951

Knewtson, Sharon, 808, 899

Knowles, N. Richard, 786, 919

Kobayashi, Hideka, 831, 985

Kobayashi*, Kent, 949

Kobayashi, Kent, 807

Koenig, Rich, 819, 887

Koenig, Rosalie, 801, 868

Kopp, Kelly, 842, 1004

Kopsell*, Dean, 788, 934

Kopsell, Dean, 806, 814, 831, 875, 986

Korban*, Schuyler, 792, 829, 843, 1007

Korban, Schuyler, 832, 993

Kornegay*, Julia, 812

Kosittrakun, Manit, 811, 882

Kosola*, Kevin, 789, 828, 896, 936

Kosola, Kevin, 798, 799, 828, 896

Kotoda*, Nobuhiro, 829, 975

Kozarewa, Iwanka, 842, 998

Kratsch, Heidi, 832, 842, 996, 1004

Krause, Charles, 809, 844, 963, 1018 
Krebs, Stephen, 829, 974

Kreider, Loretta, 789, 934

Krewer, Gerard, 797, 860

Krieger, Robert, 816, 884, 885

Krizek*, Donald, 953

Krizek, Donald, 808

Kroggel*, Mark, 786, 923

Kroggel, Mark, 944

Krueger*, William, 842, 999

Krug*, Brian, 846, 918

Krupa, James, 836, 908

Kubota*, Chieri, 801

Kubota, Chieri, 786, 831, 923, 944, 984

Kuehny*, Jeff, 889, 948

Kuehny, Jeff, 807, 820

Kulen, Oktay, 809, 958

Kumar, G.N.M., 919

Kumar, Dibyendu, 822

Kumar, G.N.M., 786

Kunitake, Hisato, 831, 986

Kurtural*, S. Kaan, 797, 858

Kurtural, S. Kaan, 797, 809, 857, 960

Kusakabe*, Ayako, 800, 863

Kwon, Youn Jung, 807, 947

\section{$\mathbf{L}$}

LaBonte, Don, 786, 819, 843, 886, 922, 1007

Lacey, Ronald E., 832, 994

Lada*, Rajasekaran R., 811, 843, 1010

Lada, Rajasekaran R., 788, 879, 928

Lakso, Alan, 833, 903

Lam, Frankie, 788, 932

Lamb, Elizabeth, 805

Lamberts*, Mary, 819, 886

Lamberts, Mary, 785

Lambrinos, John, 790, 850

LaMolinare*, Bubba, 843, 1012

LaMolinare, Bubba, 802

Lamont*, Jr., William J., 837

Lamont, Jr., William J., 801, 867

Lampinen, Bruce, 798

Landes, Reid D., 828, 899

Lang*, Gregory, 811, 838, 881

Lang*, Suzanne, 831, 990

Laraway, Debra, 830, 981

Larco*, Handell, 828

Larco, Handell, 897
Larsen, Stuart, 831, 992

Laughlin, Wanda, 801, 868

Layne*, Desmond, 842, 998

Layne, Desmond, 790

Lazur, Andy, 942

Lea-Cox*, John, 828, 898

Lea-Cox, John, 833, 902

Leap, Jim, 808, 952

Leatherwood*, W. Roland, 820, 889, 890

Lee*, Chiwon W., 819, 887

Lee*, Dong Hyuk, 909

Lee, Dong Hyuk, 836

Lee*, Eunkyung, 788

Lee, Eunkyung, 927

Lee*, Hyunju, 787, 926

Lee*, Jae Wook, 869

Lee, Jae Wook, 786, 805, 806, 872, 923

Lee, Je-min, 926

Lee*, Jin Su, 830, 981

Lee, Jin Su, 808, 955

Lee, Jong Nam, 844, 1020

Lee*, Jung Sup, 871

Lee, Jung Sup, 806

Lee, Kyung Hwa, 846, 916

Lee, Nean, 786, 807, 832, 919, 948, 992

Lee, Sang Gyu, 819, 887

Lee, Sang Kyu, 808, 955

Lee*, Seung Koo, 787, 927

Lee, Seong Koo, 788, 844, 926, 927, 981,1020

Lee, Sin Ho, 916

Lee, Si Young, 786, 923

Lee, Soo-Seong, 796, 856

Lee, Soon Won, 909

Lee, Sung Eun, 887

Lee, Yong Beom, 819, 887

Lee, Young Ran, 846, 917

Lefsrud*, Mark, 875

Lefsrud, Mark, 806

Lehrer*, Jonathan, 832, 901

Lehrer, Jonathan, 846, 915

Leinauer*, Bernd, 814

Leinfelder*, Michelle, 842, 845, 912, 1000

Leite*, Roberto de Aquino, 789, 936

León, Lorenzo, 827, 895

Leskovar*, Daniel, 811

Leskovar, Daniel, 811, 842, 843, 879, 1002,1013

Lesser, Katheryn, 842, 1001
Lester*, Gene, 844, 1019

Lester, Gene, 788, 789, 934, 937

Levasseur, Virginie, 805

Levi*, Amnon, 843, 1012

Levi, Amnon, 802

Levy, Yoseph, 800, 864

Lewers, Kim S., 844, 1022

Lewis, Kathy, 791

Leyva-Estrada, Norma, 829, 974

$\mathrm{Li}^{*}, \mathrm{Li}, 792$

Li*, Qiansheng, 846

Li, Jianguo, 836, 908

Li, Qiansheng, 917

Li, Weijie, 951

$\mathrm{Li}^{*}$, Yonghao, 829, 971

Li, Yuncong, 801, 868

Li, Zhijian, 797, 858

Liang, Wenbin, 819, 888

Liddycoat*, Scott, 808, 955

Liedl*, Barbara E., 791, 796, 820, 855, 891

Liedl, Barbara, 786, 788, 807, 808, 826

Light, Heather, 807, 947

Lillard*, Patrick, 842, 1001

Lin*, Wayne, 844, 1018

Lindgren*, Dale, 831, 987

Lindstrom, Jon, 832, 900

Lindstrom, Thor, 943

Lineberger, R. Daniel, 815, 820, 892, 997

Ling, Kai-shu, 843, 1012

Liu, Miao, 807, 948

Liu, Weizhong, 800, 861

Liu, Wenge, 802, 843, 1013

Liu*, Xiaoyu, 843, 1007

Lizana*, Antonio, 830, 981

Llewellyn, Jennifer, 800, 861

Lloyd, John, 842, 1003

Lluch*, Yolanda, 871

Lluch, Yolanda, 806

Lobato-Ortiz*, Ricardo, 844, 1015

Lobos*, Gustavo, 800, 864

Locascio, Sal. J., 820

Locke, James, 809, 844, 963, 1018

Lombardini*, Leonardo, 968

Lombardini, Leonardo, 810, 845, 911

López*, Jesús, 809, 962

López-Aguirre, Gerardo, 789, 937

Lovatt*, Carol J., 786, 830, 920, 979

Love*, Stephen, 810, 968 
Lowe, Jeremiah, 789, 939

Lu, Guiping, 809, 959

Lu, Zhongge (Cindy), 788, 929

Luan, Feishi, 796, 856

Lubag-Arquiza*, Amihan, 829, 974

Lubell*, Jessica, 846, 915

Lubell, Jessica, 832, 901

Luby*, James, 826, 1008

Luchsinger*, Luis, 830, 981

Lumpkin*, Thomas, 804

Ly, Siov Bouy, 831, 991

Lynch*, Sean, 797, 857

Lyons*, Robert, 836

Lyons, Robert, 788, 790, 810, 827, 839, $852,853,894,929,967$

\section{M}

Ma*, Hongmei, 829, 971

Ma, Lena, 801, 866

Macarisin, Dumitru, 832, 993

Macchiavelli*, Sofia, 844, 1019

Macchiavelli, Raul, 1019

MacDonald, Robert, 832, 995

Machado, Rui, 844, 1021

Macias-Duarte*, Rubén, 843

Macias-Duarte, Rubén, 809, 960, 1009

Macías-Duarte, Rubén, 786, 808, 924 , 955

Mackay*, Wayne, 1016

Mackay, Wayne, 807, 844, 845, 876, 911

Madero-Tamargo, Joaquín, 843, 1008

Maezawa, Shigenori, 787, 927

Majcherek, Tamara, 820, 891

Makk, Jose, 814

Makus*, Donald, 789, 937

Maletta, Martha, 836, 842, 908, 999

Malik, Aman Ullah, 835, 845, 906, 913

Malik, Saeed Ahmad, 845, 913

Malladi*, Anish, 801, 866

Mangiafico, Salvatore, 789, 940

Manyamgari*, Pratap, 835, 906

Maranto, Joseph, 808, 957

Marchant*, Gary E., 792

Margosan, Dennis, 830, 980

Markhart*, Albert, 875

Markhart, Albert, 806

Marsh*, Lurline, 789, 938

Marsh, Lurline, 789, 832, 836, 938

Marshall*, Donna, 844, 1020
Marti, Joan Tous, 845, 912

Martin*, Chris, 790, 824, 853

Martin, Ian F., 797, 857

Martin, Jr., Robert, 844, 1021

Martin-Duvall, Tome, 831, 991

Martinez, Miriam, 844, 1014

Maruhnich, Stefanie, 822

Mason*, Shannon, 820, 892

Massa*, Gioia, 822

Massa, Gioia, 943

Matan, Eli, 790, 851

Mathers*, Hannah, 997

Mathers, Hannah, 786, 791, 806, 832, $841,854,876,921,995$

Matsumoto, Katsuhiro, 811, 880

Mattheis, Jim, 830, 981

Matthews, Ben, 822

Maul, Pilar, 830, 982

Max, Shane, 842, 1000

Mayhew, Kelly, 829, 972

Mayland, Paul G., 788, 932

Mazalewski, Robert, 820, 891

McAfee*, Jason, 831, 991

McAngus, Andrews, 810, 966

McAvoy, Eugene, 811, 879

McCall, Ingram, 846, 918

McCollum*, Greg, 830, 981

McCoy, Lashelle, 786, 922

McCreight*, James D., 843, 1013

McCreight, James D., 843, 1013

McCrimmon, James, 854

McCullough, Deb, 810, 878

McCune*, Letitia, 826

McDonald*, Garry V., 828, 898

McDonald, Garry V., 828, 898

McDonald*, Mary Ruth, 788, 933

McElroy*, J. Scott, 815

McElroy, Scott, 788, 934

McFarland*, Amy, 945

McFarland, Amy, 807

McFerson*, Jim, 812

McFerson, James R., 836, 907

McFerson, Jim, 810, 969

McGiffen, Milton, 790, 796, 801, 852, 867

McGinnis*, Esther E., 788, 930

McGranahan*, Gale, 798

McGrath*, C.J., 952

McGrath, C.J., 808

McGregor*, Cecilia, 786, 922

McIntosh, Dennis, 942
McKay*, Steven J., 1008

McKenney*, Cynthia, 832, 844, 900

McKenney, Cynthia, 832, 900, 1016

McKenzie*, Cindy, 872

McKenzie, Cindy, 806

McKeown, Alan, 788, 808, 933, 957

McLean*, Gail, 826

McLemore, William, 819, 886

McMahan*, Linda, 810, 820, 853, 892, 967

McMahan, Linda, 790

McMahon*, Peg, 836

McNamara, Steve, 831, 986

Mecham, Kathi, 854

Medina*, L. Carolina, 793

Mehboob-ur-Rahman, 845

Melgar*, Juan Carlos, 800, 863

Memmott, Frederic D., 855

Mentreddy, S.R., 789, 937

Merhaut*, Donald, 789, 940

Merhaut, Donald, 937

Mersino, Edwin, 807, 949

Metcalfe, Leigh, 810, 967

Meyer, Mary, 845, 910

Meyer, Mary Hockenberry, 788, 930

Miano, Douglas, 786, 922

Michel-Rosales*, Arnoldo, 789, 935, 937

Micke, Warren, 798

Mickelbart*, Michael, 831, 832, 992, 994

Mickler*, Jan, 788, 932

Mikkelsen*, Robert, 801, 868

Miles*, Carol, 828, 899

Miles, Carol, 819, 828, 887, 899

Miller*, Chad T., 829, 973

Miller*, Jason, 942

Miller, A. Raymond, 831, 985

Miller, William, 787, 846, 917, 926

Miller, William B., 820, 829, 844, 890, 973, 1016

Million, Jeff, 840

Mimida, Naozumi, 829, 975

Mitcham, Elizabeth, 811, 882, 883

Mitchell*, Jeanmarie, 835, 907

Mitchell, Cary, 943

Mmbaga, Margaret, 829, 971

Mochizuki*, Maren, 808, 873, 953

Mochizuki, Maren, 806, 810, 830, 878, 978

Molina-Ochoa*, Jaime, 788, 931 
Molina-Ochoa, Jaime, 809, 843, 958, 1011

Momotaz*, Aliya, 844, 1015

Montri, Adam, 838

Moon, Wanki, 797, 820, 857, 892

Moore*, Jonathan, 812

Moore*, Kimberly, 844, 1018

Moore-Kucera*, Jennifer, 797, 859

Moore-Kucera, Jennifer, 831, 991

Moradinezhad*, Farid, 818

Morales-Payan*, J. Pablo, 808, 816, $817,827,830,841,954,980,996$

Morales-Payan, J. Pablo, 816, 819, 826

Moran, Renae, 830, 983

Moreau, Debra, 806, 870

Morelock, Teddy, 845, 913

Morgan*, Kelly T., 792

Morgan, John, 827, 895

Morgan, Kelly, 792, 800, 807, 811, 819, 879, 886, 946

Morgan, Robin, 790, 853

Moriya, Shigeki, 829, 975

Morrow*, Robert, 821

Morse1, R., 816

Mortley*, Desmond G., 786, 922

Mortley, Desmond, 829, 976

Moser, Bruno, 841

Mota-Sanchez, David, 810, 878

Motsenbocker*, Carl, 945

Motsenbocker, Carl, 807, 808, 952

Mou*, Beiquan, 796, 856

Mou, Beiquan, 796, 802, 855

Moydell*, Edward, 790, 853

Moydell, Edward, 790, 852

Mudge*, Kenneth W., 841

Mulholland, Nancy, 807, 947

Muller, Keona, 832, 995

Muramoto, Joji, 808, 952

Murphy, Kevin, 814

Murr, Dennis, 830, 980

Murthy*, Kotamballi N. Chidambara, 819,887

Muse*, Barbara, 786, 923

Muse*, Ronald, 786

Mutch, Dale R., 843, 1009

Mutschler, Martha A., 844, 1015

Myers*, James, 813

Myers, James, 796, 856

Myers, Stephen, 799

Myrold, David, 831, 991
$\mathbf{N}$

Nachabe, Mahmood, 829, 842, 977 , 1005

Nadal-Medina, Maria-Rocio, 843, 1008

Nadler, Arie, 809, 964

Nagata, Russell, 819, 886

Naik1, Dhananjay, 822

Nair*, Ajay, 843, 1009

Nair, Muralee, 831, 990

Nakano, Kohei, 787, 927

Nam, Chun Woo, 844, 1020

Nanthachai*, Nunchanok, 811, 882

Narciewicz, Sophia, 842, 1004

Narciso, J., 818

Narciso, Jan, 844, 1018

Natarajan*, Seenivasan, 801, 866

Nath, Prem, 809, 959

Navarro-Tarazaga*, Maria-Llanos, 869

Navarro-Tarazaga, Maria-Llanos, 805

Nawaz, Muhammad Azhar, 800, 864

Neal*, Catherine, 800, 862

Neilsen*, Denise, 812

Neilsen, Gerry, 812

Nelson*, Paul, 874

Nelson, Liz, 828, 899

Nelson, Paul, 806, 820, 890

Nemali*, Krishna, 1003

Nemali, Krishna, 790, 842849

Nesbitt*, Monte, 830, 979

Neves, Julio Cesar Lima, 789, 936

Newman*, Julie, 789

Newman, Julie, 940

Ngouajio*, Mathieu, 790, 850

Ngouajio, Mathieu, 790, 843, 850, 1009

Niederholzer*, Franz, 789, 934

Niederholzer, Franz, 842, 999

Niedziela, Carl, 787, 925

Niedziela Jr., Carl E., 788, 929

Niemiera*, Alex, 950

Niemiera, Alex, 807, 842, 1005

Nienhuis*, James, 855

Nienhuis, James, 796

Ning, Lin, 843, 1006

Nishiguchi, Mitsuru, 829, 975

Nishiyama, Kazuo, 831, 986

Niu*, Genhua, 829, 840, 845, 876, 911, 978

Niu, Genhua, 807

Njuguna*, Wambui, 846, 915

Njuguna, Joseph, 845, 913
Nock, Jacqueline, 811, 882

Noh, Hee Young, 790, 942

Nolte*, Kurt, 801, 865, 873

Nolte, Kurt, 790, 806, 808, 854, 954

Nonnecke*, Gail, 945

Nonnecke, Gail, 807, 809, 830, 960, 983

Norcini, Jeffrey G., 845, 910

Norelli, John, 832, 993

Norikane, Joey, 789, 940

Norrie, Jeff, 839

Nosarzewski, Marta, 836, 908

Nunes, Jeremy, 842, 999

Nunez-Elisea*, Roberto, 845, 912

Núñez-Ramirez*, Fidel, 787

Núñez-Ramírez, Fidel, 786, 924

Nyirakabibi, Isabelle, 789, 936

Nzokou, Pascal, 800, 810, 861, 965

\section{$\mathbf{O}$}

O'Callaghan*, Angela, 944

O'Callaghan, Angela, 790, 807, 834, 853

O’Hare, Tim, 818

Obaa, Bernard, 807, 945

Obenland*, David, 830, 980

Obreza, Thomas, 793

Obreza, Tom, 811, 879

Offir, Yossi, 790, 851

Ogata, Jun, 829, 971

Ogbuchiekwe, Eddie, 801, 867

Ogden*, Andrew, 797, 860

Ogden, Elizabeth L., 823

Ognjanov, Vladislav, 843, 1007

Ogutu*, Maurice, 788, 933

Ogutu*, Rose, 789, 935

Oh*, Jiyoung, 808, 956

Oh*, Myung-Min, 832, 993

Oh, Dae-Geun, 833, 904

Oh, Joung-Youl, 829, 975

Ohkoshi, Takahiro, 802

Oki*, Loren, 820, 845, 891, 911

Olczyk, Teresa, 801, 868

Oleksak*, Brian, 810, 968

Olsen*, Jeff, 804

Olsen, Jeff, 804, 826

Olsen, Nora, 787, 926

Olsen*, Richard T., 831, 987

Ortega, Daniel, 807, 944

Ortega, Lisa, 944 
Orvis*, Kathryn, 836, 947

Orvis, Kathryn, 807, 828

Orzolek, Michael D., 801, 867

Osborne, Lance, 790, 801, 852, 868

Osuna-Castro, Juan Alberto, 789, 935

Ott*, Kristy, 819, 887

Ouellette, David, 810, 970

Owen*, Jim, 839

Owens, Christopher, 806, 809, 961

Owings, Al, 810, 876

Ownley, B.H., 788, 933

Ozgen, Mustafa, 831, 985

Ozores-Hampton*, Monica, 811, 879

Ozores-Hampton, Monica, 807, 811, 880, 946

\section{P}

Pacumbaba, Rudy, 805, 870

Padhye*, Sonali, 846, 916, 948

Padhye, Sonali, 807

Padley, Jr.*, Les, 843, 844, 1014

Painton*, Michael, 854

Pak, Chun Ho, 819, 846, 887, 917

Palaniswamy*, Usha Rani, 809, 959

Palumbo*, Rose, 809, 963

Pan*, Jhen-Ying, 786

Pan, Jhen-Ying, 919

Pantalone, Vince R., 832, 900

Panter, Karen, 785, 828, 837, 846

Papenfuss*, Kylara A., 943

Parfitt*, Dan, 808, 831, 957, 991

Parfitt, Dan, 808, 829, 957, 976

Park*, Sin-Ae, 946

Park*, Soon, 809, 843, 961, 962, 963, 1006

Park*, Suhyoung, 796, 856

Park, Hyo-Guen, 796, 856

Park, Jong Han, 806, 872

Park, Kyoung Sub, 805, 869

Park, Sin-Ae, 807

Park, Sunghun, 833, 904

Parmenter*, Darrin, 819, 886

Parmenter, Darrin, 811, 879

Parsons*, Eugene, 829, 975

Parsons, Lawrence, 846, 917

Patil*, Jaiparkash R., 800, 863

Patil, Bhimanagouda, 796, 800, 816, $819,828,829,831,835,863,885$, 887, 985, 976, 997

Patil, Bhimu S., 831, 986
Pavlis, Mike, 829, 978

Paz, Maria, 809, 965

Pearson*, Brian, 809, 964

Pemberton*, H. Brent, 787, 789, 925, 939

Pemberton, H. Brent, 845, 911

Peña-Beltrán, Elpidio, 789, 935

Peng, Zhonghua, 788, 931

Pennisi, Bodie, 826

Percivalle, Cynthia, 842, 1004

Perez*, Jose Luis, 831, 985

Pérez, Juan Miguel, 843, 1008

Perez, Kari W., 805

Perez-Gago, Maria-Bernadita, 805, 869

Perez-Perez, J.G., 790, 849

Perkins-Veazie*, Penelope, 788, 801, 809, 840, 844, 927, 959, 1020

Perkins-Veazie, Penelope, 789, 802, 934

Perry*, Ronald, 859

Perry, Ronald, 797, 820, 859

Pesis*, Edna, 811, 882

Peterson*, Allison, 947

Peterson, Allison, 807

Petracek, Peter, 820, 840

Pfeffer*, Jenny, 946

Pfeffer, Jenny, 807

Pharr, Kathryn, 829, 972

Phatak, S.C., 816

Philip, Elizabeth, 789, 938

Pierzynski, Gary, 789, 935

Pike, Leonard M. 819, 887

Pillai, Suresh D., 829, 976

Pineda-Pineda, Joel, 789, 936

Pittcock*, J.K., 842, 1001

Pittenger, Dennis R., 978, 830

Plotto, A., 818

Plotto*, Anne, 844, 869, 1018

Plotto, Anne, 805, 844, 1018

Podaras, Peter, 841

Poland, Therese, 810, 878

Polashock, James, 830, 982

Poling*, Barclay, 823

Poling, Barclay, 794, 795, 796

Polito, Vito, 801, 865

Pomper*, Kirk, 789, 809, 831, 939, 960, 985

Pomper, Kirk, 809, 811, 844, 881, 1019

Pooler*, Margaret, 829, 971

Pop, Ioana, 951

Pop, Rodica, 807, 829, 951, 972

Porat, Ron, 830, 982
Poritz, Darwin, 786, 922

Portz*, Dennis, 830, 983

Posalski, Itzhak, 790, 851

Poss, James, 842, 1002

Pottorff*, Laura, 839

Powell*, W.A., 788, 933

Powers, Joe, 819, 887

Pozo, Luis, 806, 871

Pozueta, Javier, 919

Pozueta, Javier, 786

Prasad, Amikha, 818

Prehn, Alison, 839

Prestowitz*, Catherine, 788, 929

Price*, David L., 855

Prins, B., 833

Pritts, Marvin, 828, 830, 897, 983

Proano*, Alvaro, 842, 1002

Puangchit, Ladawan, 845

Pulam, Taweesak, 797, 857

R

Raba, Richard, 833, 903

Rabah, Isselmou Ould, 829, 971

Radcliffe, David, 842, 1003

Rademacher*, Janet, 817

Rader, Heidi, 787, 924

Radillo-Juarez*, Francisco, 809, 843, 1011

Radillo-Juarez, Francisco, 842, 931, 1000

Radillo-Juárez, Francisco, 789, 935

Rafie*, Ray, 886

Rafie, Ray, 819

Rahman, Mehboob-ur, 913

Rajapakse*, Nihal, 786, 923

Rajasekaran, Kanniah, 789, 941

Rajashekar, Channa, 832, 993

Rallo*, Luis, 827, 831, 895, 988

Ramos, Leandro, 844, 1014

Ramsay, Gavin, 825

Rangarajan*, Anusuya, 801, 867

Rangarajan, Anusuya, 806, 873

Rao, Sheetal, 832, 994

Rapaka*, Vijaya, 820, 889

Rapoport, Hava F., 790, 850

Ratanachinakorn, Benjamas, 811, 882

Ratnayaka*, Harish, 805

Ratnayaka, Harish, 816, 943

Ratner, Kira, 790

Raveh*, Eran, 800, 809, 864, 964 
Ray*, Dennis T., 817

Ray, Dennis, 785, 807

Rayirath*, Usha P., 788, 928

Read, Paul, 785, 809, 959

Reddy, Amarender S., 835, 906

Reddy, Umesh O.U., 843, 1012

Reed, Barbara, 846, 914

Reed, David W., 832, 994

Reed, Jenn, 828, 899

Reed, Sandra, 809, 829, 832, 900, 964, 971

Reese, R. Neil, 831, 985

Regan, Richard, 789, 939

Reganold, John, 819, 887

Reginato, Gabino. 845, 914

Reichard, Katie. 810, 831, 969, 992

Reighard*, Gregory, 810, 843, 970, 1007

Reilley, Ann, 827, 895

Reilly, Jackie, 810, 967

Reiners, Stephen, 843, 1010

Renquist, Steve, 790, 853

Retamales, Jorge, 800, 864

Reuss*, Rachel, 809, 959

Revankar, Sachit, 832, 994

Reyes-Martínez, Celso, 789, 935

Reynolds*, Robert, 810, 965

Reynolds, Robert, 790, 849

Richard, Mike, 810, 876

Richards, Christopher, 827, 895

Richards, Jeffrey, 806, 875

Richart, Gregory, 806, 876

Righetti*, Timothy, 790, 850, 858

Righetti, Timothy, 797

Rinehart, Timothy (Tim), 829, 832, 900, 970, 971

Riseman, Andrew, 835

Ristvey*, Andrew, 833, 902

Ristvey, Andrew, 828, 898

Rivera-Montelón, Mireya, 788, 931

Robacker, Carol, 829, 970

Robbins, LeRon, 833

Roberson, William R., 787, 789, 925, 939

Roberts*, Glenn, 831, 990

Roberts, Pamela, 843, 844, 1014

Robinson*, Carolyn W., 827, 894

Robinson*, Terence, 845, 914

Robinson, Terence, 833

Robles-Contreras*, Fabián, 808, 809, 955, 960
Robles-Contreras, Fabián, 786, 809, 843, 924, 1009

Rodermel, Steven, 789, 934

Rodriguez, Denise, 807, 829, 845, 876, 911, 978

Rodriguez, Luis, 828, 898

Rodríguez-Landero, Ana del Carmen, 843,1008

Roe*, Nancy, 886

Roe, Nancy, 819

Rogers, John, 831, 990

Roka, Fritz, 811, 879

Rom*, Curt, 836

Rom, Curt, 831, 991, 992

Romelczyk, Stephanie, 796

Romer*, James, 820, 891

Romero, Agusti, 845, 912

Romo*, Josefina, 809, 962

Roper*, Teryl, 828, 897

Roper, Teryl, 828, 896

De la Rosa, Raúl, 895, 988

Rosen, Carl J., 794

Rosenstock*, Todd, 833, 902

Ross, David, 828, 898

Ross*, Matthew, 950

Ross, Matthew, 807

Rouse, R.E., 842, 999

Rouseff*, Russell L., 840

Rowe*, Bradley, 824, 842, 1003

Rowland*, Lisa J., 823

Rowland, Lisa J., 809, 963

Rowley*, Laura, 943

Ruberson, J., 816

Rubino, Maria, 816, 884

Rudell*, David, 830, 981

Rufty, Thomas, 820, 890

Rukuni*, Dzingai, 845, 910

Rumpho, Mary, 810, 966

Runkle, Erik, 799, 807, 844, 948, 1018

Ruter, John, 828, 898

\section{S}

Sabbatini*, Paolo, 800

Sabbatini, Paolo, 800, 835, 864, 907

Saftner*, Robert, 830, 982

Saftner, Robert, 844, 1019

Saha*, Shubin, 788, 932

Saha, Uttam, 801, 866

Sakaguchi, Yoshihisa, 829, 976

Sakai, Miho, 831, 986
Sakata, Yusuke, 971

Sakata, Yusuke, 829

Sakhanokho*, Hamidou F., 789, 941

Salaiz, Thomas, 810, 968

Salandanan*, Karen, 809, 958

Saltveit*, Mikal, 870

Saltveit, Mikal, 805

Sambo, Paolo, 829, 973

Sams*, Carl, 786, 921

Sams, Carl, 785, 788, 806, 807, 809, $831,875,934,961,986$

Sanchez*, Charles, 816, 884, 885

Sanchez, Charles A., 808, 954

Sanchez*, Elsa, 801, 867

Sanchez, Elsa, 808, 830, 844, 953, 983, 1021

Sanchez-Arevalo, Emilio, 830, 979

Sanders*, Graham, 844, 1021

Sandrock, David, 790, 809, 850, 965

Sankhla, Narendra, 844, 1016

Santos*, Kathryn, 829, 974

Sapir*, Gal, 833, 903

Sargent, Steve, 801, 868

Sargent*, Steven, 788, 928

Sargent, Steven, 835, 907

Sartain, Jerry B., 793

Sato*, Makiko, 831, 986

Saunders, Kevin, 807, 945

Saxena*, Ashish, 844, 1015

Scagel, Carolyn, 789, 939

Schaaf, Daniel, 831, 987

Scheerens*, Joseph C., 831, 987

Scheerens, Joseph, 831, 985

Scheiber*, Michele, 809, 964

Scheiber*, Sloane, 809, 965

Scheiber, Michele, 845, 847

Schloemann, Sonia, 830, 984

Schluttenhofer*, Craig, 943

Schmidt*, Tory, 810, 969

Schmitt, William, 828, 897

Schnelle*, Mike, 844, 1017

Schrader, Larry, 836, 909

Schreiber*, Henry, 829, 972

Schreiner*, R. Paul, 797, 857

Schroeder, Michael, 835, 907

Schuch*, Ursula K., 789, 940

Schumann*, Arnold, 800, 862

Schumann, Arnold W., 800, 862

Schupp*, James, 831, 992

Schupp*, James R., 842, 1001

Schupp*, Jim, 810, 969 
Schuster, David, 844, 1015

Schuster, Todd, 843, 1010

Schutzki, Robert, 807, 950

Schwallier, Philip G., 835, 907

Sciarappa*, William, 797, 860

Sciarappa, William, 796, 809, 958

Scoffield, Jessica, 829, 976

Scorza*, Ralph, 833, 904

Scott*, John, 844, 1015

Scott, John, 855

Scott*, Samuel, 886

Scott, Samuel, 819

Scurlock, David, 809, 961

Seavert, Clark, 833, 842, 902, 1000

Sedgley, Margaret, 818

Seiler, John, 829, 978

Semmens, Kenneth, 831, 942, 988

Senesac, Andrew, 823

Seo, Sang Tae, 806, 872

Seong, Ki Cheol, 830, 981

Serdani, Maryna, 830, 981

Seymour, Kerry, 810, 967

Shafir, Sharoni, 833, 904

Shahak*, Yosepha, 790, 851

Sharber*, Casey, 790, 852

Sharma*, Jyotsna, 840

Shaw*, Nicole, 790, 852

Shaw, Nicole, 788, 928

Shaw, Ruth G., 1008

Sheely*, Deborah, 826

Sheely, Deborah, 833

Shen*, Xiang, 829, 978

Shennan, Carol, 808, 952

Sherwin, Jack, 829, 976

Shigematsu*, Kenta, 802

Shigki, Thoshiro, 833, 904

Shimizu, Keiichi, 829, 971

Shin, Hak Ki, 820, 890

Shin, Il-Sheob, 845, 913

Shin, Yong-Uk, 845, 913

Shinkle, Jim, 943

Shinohara*, Togo, 811, 879

Shoemaker*, Candice, 827, 893

Shoemaker, Candice, 807, 946

Shrefler*, James, 844, 1020

Shrestha, Surendra, 842, 998

Shrestha, Surendra Lal, 844, 1014

Shukla, Sanjay, 811, 879

Sibley, Kevin, 928

Sibley, Jeff, 800, 845, 861, 911
Sibley, Kevin, 788

Sideman, Eric, 808, 951

Sievert, Jim, 830, 980

Silvernail, Anthony, 808, 831, 954, 988

Silvoy, J., 816

Simmon, Philipp, 843, 1013

Simon, Jim, 797, 860

Simonne, Amarat, 788, 928

Simonne*, Eric, 811, 879

Simonne, Eric, 801, 811, 868, 879

Sims*, Cedric, 789, 937

Singh, Anand Kumar, 846

Singh, Bharat, 808, 843, 954, 1011

Singleton*, Mary, 808, 955

Sisson, Jeremy M., 796, 820, 855, 891

Skinner, John A., 832, 900

Skogerboe, D., 809, 963

Slaughter, David, 830, 980

Smagula*, John M., 789, 934

Smalley*, Timothy, 827, 894

Smalley, Tim, 827, 893

Smith, Alan, 791

Smith, Alan G., 799

Smith*, Brandon, 809, 961

Smith, Durward, 809, 959

Smith*, Erick, 811, 880

Smith, Leanna, 807, 945

Smith, Melissa, 796, 820, 855, 891

Smith*, Michael, 808, 956

Smith*, Nichole, 831, 988

Smith, Powell, 827, 895

Smith*, Richard, 952

Smith, Richard, 794, 808, 828, 900

Smithyman, Russell, 797, 857

Snapp, Sieglinde S., 843, 1009

Somireddy*, Upender, 786, 921

Sommeechai, Montathip, 845

Sommerfeld* , Aime, 946

Sommerfeld, Aime, 807

Son, Dong-Soo, 845, 913

Son, Ki-Cheol, 807, 947

Song*, Jun, 870, 871

Song, Jang-Hoon, 845, 913

Song, Jun, 806

Sorochan*, John, 815

Sosa*, Sergio, 820, 889

Soundy, Puffy, 789, 939

Spada, Piero A., 828, 896

Spann*, Timothy, 811, 881

Spayd, Sara, 794
Spayd, Sara E., 795

Spears, Karen, 810, 967

Spiers*, Jay, 833, 902

Spiers, James, 829, 844, 971, 1020

Spotts, Robert, 830, 981

Sprenkel, Richard, 801, 868

Singh, Sr., Anand Kumar, 915

Sriladda, Chalita, 845

Srmack, William, 805, 869

St. Hilaire*, Rolston, 832, 842, 993, 1002

St. Hilaire, Rolston, 789, 790, 829

Staats, David, 810, 877

Staats, E., 809, 963

Stack*, Lois B., 810, 966

Stack, Lois B., 787, 925, 806, 874

Stackpoole*, Sarah, 789

Stackpoole, Sarah, 936

Stamps*, Robert, 842, 917, 1004

Stamps, Robert, 801, 846, 866

Stansly, Phil, 811, 879

Stansly, Philip A., 786, 921

Starman, Terri W., 820, 833, 846, 892, 902, 916

Staub*, Jack, 796, 856

Staub, Jack, 843, 846, 915, 1013

Steeves, Cody, 833, 903

Stefanelli*, Dario, 797, 859

Stefanelli, Dario, 797, 859

Steil*, Aaron, 810, 967

Steinberg, Susan, 785

Stern, Raphael (Raffi), 833, 904

Stevens, Mikel R., 855

Stewart*, J. Ryan, 828, 899

Stewart, Curtis, 807, 946

Steyn, J. Martin, 789, 939

Stier*, John, 815

Stinner, Deborah, 797, 860

Stoffella, Peter, 790, 832, 835, 852, 907, 996

Stommel*, John, 843, 1006

Stonaker, Frank, 958

Stonaker, Frank, 809

Stover*, Ed, 833

Stover, Ed, 820, 822, 835

Strachan, Janice, 799, 837

Strahan, Ron, 810, 877

Stratton*, Jayne, 809, 959

Stratton, Jayne, 809, 959

Strik, Bernadine, 790, 828, 850, 897 
Strik, Bernadine C., 844, 1021, 1022

Stringer, Stephen, 844, 1020

Struve, Daniel, 828, 899

Struve, Daniel K., 831, 987

Studstill, David, 811, 880

Stushnoff, Cecil, 809, 958

Stushnoff, Jeannette, 809, 958

Stutte*, Gary, 875

Stutte, Gary, 806

Suazo*, Gerardo J., 820

Suazo, Gerardo J., 890

Subbarao, Krishna, 828, 896

Sudal, June, 843, 1009

Sugimoto*, Nobuko, 869

Sugimoto, Nobuko, 805

Sugimoto, Yasuhiro, 831, 986

Sullivan, Dana, 817

Sullivan, J. Alan, 831, 987

Sun*, Youping, 787, 925

Sun, Feng-Jie, 829

Sun, Feng-jie, 975

Sutter, Michelle, 801, 868

Swift*, Curtis, 830, 979

Swire-Clark, Ginger, 843, 1007

Syvertsen*, Jim, 790, 849

Syvertsen, James P., 800, 863

Syvertsen, Jim, 790, 849

\section{T}

Taber*, Henry, 934

Taber, Henry, 789

Tadmor, Yaacov (Kobi), 843, 1012

Takahashi, Sae, 829, 975

Takaki, Ryoko, 831, 986

Takeda*, Fumiomi, 801, 865

Takeda, Fumiomi, 800, 844, 864, 1022

Talbot, Timothy, 819, 886

Tamura, Fumio, 811, 880

Tanabe, Kenji, 811, 880

Tanck, Sandy, 807, 947

Tanis*, Sara, 810, 878

Tarara, Julie, 797, 857

Tay, David, 809, 841, 845, 910, 963, 997

Taylor, Alan G., 844, 1016

Taylor, Bradley H., 797, 820, 857, 892

Taylor*, Matthew, 820, 874, 890

Taylor, Matthew, 806, 820, 889

Taylor, Merritt, 844, 1020
Taylor, Milton, 842, 1003

Teasdale, John, 808, 953

Techavuthipron*, Chairat, 787, 927

Telias*, Adriana, 842, 999

Tetsumura, Takuya, 831, 986

Tetteh*, Antonia, 827, 896

Thetford*, Mack, 841

Thies, Janice, 801, 867

Thomas*, Elizabeth J., 786, 921

Thompson*, Ellen, 844, 1021

Thompson, Anthony Keth, 835, 905

Thompson, Jim, 830, 980

Thomson, Cynthia, 786, 923

Thro*, Ann Marie, 821, 846, 914

Tian*, Daike, 845, 911

Tichy, Shane, 816, 885

Tickes, Barry, 806, 873

Tilt, Ken, , 845, 911

Torbert, H. Allen, 800, 861

Trader*, Brian, 787, 925

Trader, Brian, 787, 926

Treadwell*, Danielle, 801, 868

Trewatha*, Pamela, 950

Trewatha, Pamela, 807

Trigiano*, Robert, 809, 964

Trigiano, Robert, 829, 832, 900, 970, 971

Trueman*, Cheryl, 788, 933

Tsai*, Ching-Jung, 832, 901, 948

Tsai, Chin-Jung, 806, 874

Tsai, Ching-Jung, 807

Tsuda, Hirotoshi, 831, 986

Tulio, Jr.*, Artemio, 831, 985

Turner, M., 809, 963

\section{U}

Ueno, Hideo, 808

Uhm, Jal Youl, 836, 909

Ullah*, Hameed, 835

Ullrich, Steve, 831, 989

Um, Young Cheol, 808, 955

Umeda*, Kai, 1005

Umeda, Kai, 842

\section{V}

Valdez-Aguilar*, Luis A., 842, 1002

Valdez-Aguilar, Luis-Alonso, 789, 936

Valentino, Tara, 831, 990
Valenzuela-Ruiz*, Manuel de Jesus, 809,960

Valenzuela-Ruiz, Manuel de Jesus, 786, 808, 843, 924, 955, 1009

Vallad, Gary, 828, 896

Vallejos, C.E., 811, 883

VanLeeuwen, Dawn, 789, 790, 941, 942

van Iersel*, Marc, 790, 842, 849, 889

van Iersel, Marc, 797, 820, 827, 836, 860, 894, 1003

Van Vranken, Richard, 797, 860

Vargas-Ortega, Luis Antonio, 843, 1011

Vaughn, Steven, 786, 921

Vendrame*, Wagner, 829, 974

Vendrame, Wagner, 839, 841

Verdegaal, Paul, 798

Verhey, Steven, 831, 989

Verlinden, Sven, 808, 954

Viadero, Roger, 831, 988

Vieira, Gerival, 789, 936

Vikram*, Amit, 829, 976

Villalobos*, Max, 811, 883

Villalobos, G., 830, 981

Villordon*, Arthur, 819, 843, 886, 1007

Viveros, Mario, 798

Vivo, Nuria Mallen, 845, 912

Volder*, Astrid, 810, 965

Volk*, Gayle, 827, 895

von Fraunhofer, J.A., 831

Vuppalapati, Pavani, 836, 908

Vyapari*, Sudeep, 829, 842, 977, 1005

W

Wade, Nicholas, 829, 972

Wadl*, Phillip A., 829, 832, 900, 970

Waliczek*, Tina M., 810, 968

Waliczek, Tina, 807, 945

Walker, Lloyd, 805, 870

Walker, Sonia, 797, 859

Wall*, Marisa, 830, 982

Wallon, Robert, 843, 1007

Wally*, Abdelfattah, 811, 881

Walters*, S. Alan, 820, 827, 892, 894

Walters, S. Alan, 797, 857

Wang*, Changzheng, 843, 1012

Wang, Changzheng, 831, 985

Wang*, Chien, 809, 830, 958, 980

Wang, Guo-Liang, 809, 963

Wang*, Guangyao, 801, 867

Wang, Lixue, 842, 1004 
Wang, Min, 843, 1011

Wang, Qingju, 829, 978

Wang*, Shiow, 809, 958

Wang*, Shiping, 797, 857

Wang, Shiow, 830, 980

Wang*, Xinwang, 809, 964

Wang, Xinwang, 829, 832, 900, 970

Wang*, Yin-Tung, 806, 874

Wang, Yin-Tung, 846, 916

Wang, Ying, 790, 810, 849, 965

Wang, Yiqiang, 819, 888

Warmund*, Michele, 808, 956

Warmund, Michele, 794, 795, 808, 956

Warner*, Ryan M., 949

Warner, Ryan M., 807

Warnock*, Daniel, 832, 844, 846, 901, 918, 1017

Warnock, Daniel, 787, 846, 916, 926

Warren, Stuart, 839

Warsaw*, Aaron, 789, 940

Wasilwa*, Lusike, 845, 913

Wasmer, Nick, 832, 994

Waters, Jr., Luther, 827, 894

Watkins, Christopher (Chris), 811, 830, 882, 982

Watson, Todd, 810, 965

Wechter, W. Patrick, 843, 1012

Weeda*, Sarah M., 786, 919

Wehner, Todd, 785, 808, 827, 896, 956

Wells*, Christina, 799

Wells*, Lenny, 808, 956

Wells, Lynda K., 843, 1010

Wenk, Benjamin, 842, 1001

Werner, Jeffrey, 787, 924

Werner, Les, 810, 877

Wert*, Todd, 842

Wert, Todd, 999

Wessel-Beaver*, Linda, 844, 1019

West*, Todd, 836

West, Todd, 831, 832, 840, 841, 942, 988

Westerveld*, Sean, 808, 957

Westerveld, Sean, 808, 956

Weston*, Leslie, 786, 823, 921

Wetzstein*, Hazel, 833, 905

Wetzstein, Hazel, 833, 903

Wheeler, Raymond, 806, 821

Whipker*, Brian, 812

Whipker, Brian, 787, 846, 918, 925

Whitaker, Vance M., 831, 986

White, Douglas, 830, 982
White*, Philip, 825

White*, Sarah, 842, 1003

White, Shawn, 831, 990

White, Wendy, 789, 934

Whitehead*, Wayne, 843, 954, 1011

Whitehead, Wayne, 808

Whiting, Matt, 810, 969

Whiting, Matthew, 811, 880

Whitlark, Brian, 842, 1005

Whitman, Catherine, 807, 948

Whittemore, Alan T., 831, 987

Whittet*, Kimberly, 826

Whitwell*, Ted, 786, 920

Whitwell, Ted, 842, 1003

Wiedenfeld, Bob, 809, 964

Wien*, Chris, 838

Wien*, H. Chris, 844, 1016

Wien, Hans Christian, 829, 974

Wilfong, Kristen, 796, 820, 855, 891

Wilkin*, Matt, 842, 1006

Williams, Dave, 787, 926

Williams*, Kimberly , 844, 1017

Williams, Kimberly, 789, 935, 944

Williams*, Patrick, 791

Williamson*, Jeffrey G., 842, 999

Williamson, Jeffery G., 786, 920

Wills, James, 806, 875

Wilson*, P. Chris, 840

Wilson*, Sandra, 832, 841, 995

Wilson*, L. George, 805

Wilson, L. George, 808, 841

Wilson, P. Christopher, 832, 995

Wilson, Paul, 810, 876

Windham, Alan, 829, 971

Windham, Mark, 809, 829, 832, 900, 964, 971

Wisniewski*, Michael, 795, 832, 993

Wisniewski, Michael, 806, 876

Withrow-Robinson, Brad, 820, 892

Wittenberg, Elisabeth, 944

Wolyn, David, 808, 955

Wong, Jeffrey, 944

Wong, Lung, 818

Woo, Seongmin, 833, 905

Woods, Floyd, 805, 830, 845, 870, 911, 982

Woodson*, William R. (Randy), 812

Woodson, William R. (Randy), 785, 786,832

Workmaster, Beth Ann A., 789, 828, 896,936
Worsley, Jessica, 831, 989

Worstell, Velvet L., 796, 855

Wright*, Amy, 842, 843, 1006

Wright, Amy N., 827, 894

Wright, A.N., 808, 952

Wright*, Glenn, 845, 914

Wright, Glenn, 810, 845, 912

Wszelaki*, Annette, 788, 816, 928

Wu, Anxiang, 788, 929

Wu, Laosheng, 789, 940

Wu, Min, 944

Wycislo*, Andrew, 809, 961

Wynn, Theresa, 824

X

Xi, Qingfu, 824

Xia*, Guohai1, 833, 903

Xie, Bixia, 787, 819, 888, 925, 951

Xie, Weijie Li, Bixia, 1807

Xu, Wenping, 1797, 857

Y

Yabiku, Scott, 790, 853

Yamada, Masahiko, 829, 976

Yang*, Guochen, 788, 929

Yang*, Wei Qiang, 897

Yang, Wei Qiang, 828

Yates, Marylynn, 845, 911

Yauwapaksopon*, Daraluck, 829, 971

Ye, Xia, 791

Yeager*, Thomas, 840

Yeager, Thomas, 828, 898

Yeager, Tom, 799

Yehezkel, Hanna, 790, 851

Yen*, Christine, 846, 916

Yin*, Xinhua, 833, 842, 902, 1000

Yonemori*, Keizo, 829, 976

Yoo, Dong-Lim, 844, 1020

Yoo, Kil, 809, 962

Yoo, Kil Sun, 811, 842, 879, 1002

Yoo*, Mung Hwa, 947

Yoo, Mung Hwa, 807

Yoon, Byeong Sung, 842, 998

Yoon, Moo-Kyoung, 796, 856

Yorio, Neil, 806, 875

Yoshida, Akira, 811, 880

Young, Arnett, 828, 900

Young*, Eric, 812 
Young, Robert B., 842, 1001

Yu, Jiangfan, 819, 832, 888, 995

Yuan*, Rongcai, 810, 836, 908, 969

Yukizaki, Chizuko, 831, 986

Yum, Sung Hyun, 786, 923

\section{$\mathbf{Z}$}

Zaharia, Dumitru, 807, 951

Zahreddine*, Hala, 828, 899

Zajicek, Jayne, 807, 810, 842, 945, 968, 1001

Zajicek, Jayne M., 807, 946

Zalapa, Juan E., 843, 1013

Zamora*, Everardo, 787, 843, 1011

Zamora, Everardo, 809, 831, 924, 962, 991

Zanish-Belcher, Tanya, 807, 945

Zauche, Timothy, 806, 873, 874
Zhang, Bin, 788, 931

Zhang*, Caixi, 811, 880

Zhang, Caixi, 797, 857

Zhang*, Donglin, 788, 810, 819, 832, $888,931,966,995$

Zhang, Donglin, 788, 806, 807, 845, $874,912,925,929,951$

Zhang*, Mingshu, 787, 926

Zhang, Cheng Hao, 805, 869

Zhang, Mingshu, 787, 925

Zhang, Ping, 800, 861

Zhang, Qixiang, 912

Zhang*, Riqing, 888

Zhang, Riqing, 819, 888, 929

Zhang, Shaoliang, 832, 995

Zhang, Xingping, 809, 959

Zhang, Zhen, 791

Zhang, Zuoshuang, 845, 912

Zhao*, Xin, 816, 885
Zhao, Cindy, 828, 898

Zhao, Xuemin, 832, 995

Zheljazkov*, Valtcho, 789, 819, 888, 938

Zheng, QiFa, 806, 871

Zheng, Youbin, 800, 861

Zhong, Qiuping, 832, 995

Zhu, Ling, 788, 931

Zibilske, Larry, 789, 937

Zimmerman, Thomas, 797, 858

Zinati*, Gladis, 800, 839, 862

Zinati, Gladis, 835, 906

Zlesak*, David, 846, 915

Zlesak, David, 845, 914

Zuberer, David A., 832, 994

Zurawski, Dale, 789, 940

Zuzek, Kathy, 831, 846, 915, 986

Take your place among the leaders in public horticulture

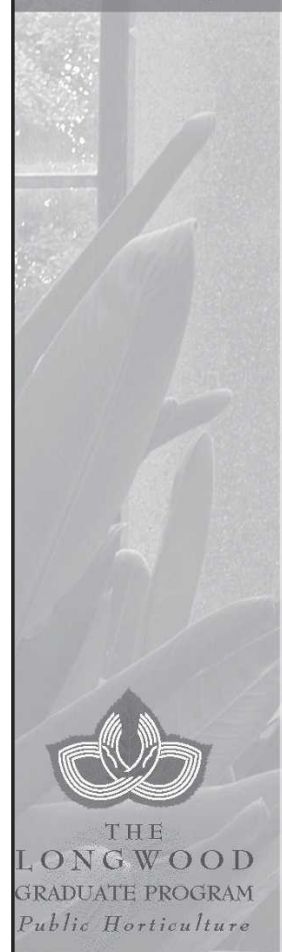

\section{Enroll in}

the Longwood Graduate Program in Public Horticulture at the University of Delaware

Blend two of the best with Longwood Gardens as your laboratory and University of Delaware as your academic home

- Experience public horticulture venues from the local to the international

- Receive a generous stipend and full tuition support

- Join the ranks of an exceptional network of former Longwood Fellows

For details about this extraordinary educational experience, visit www.udel.edu/longwoodgrad or call 302-831-2517. 


\section{Justin Hancock is a CPH ${ }^{\star}$}

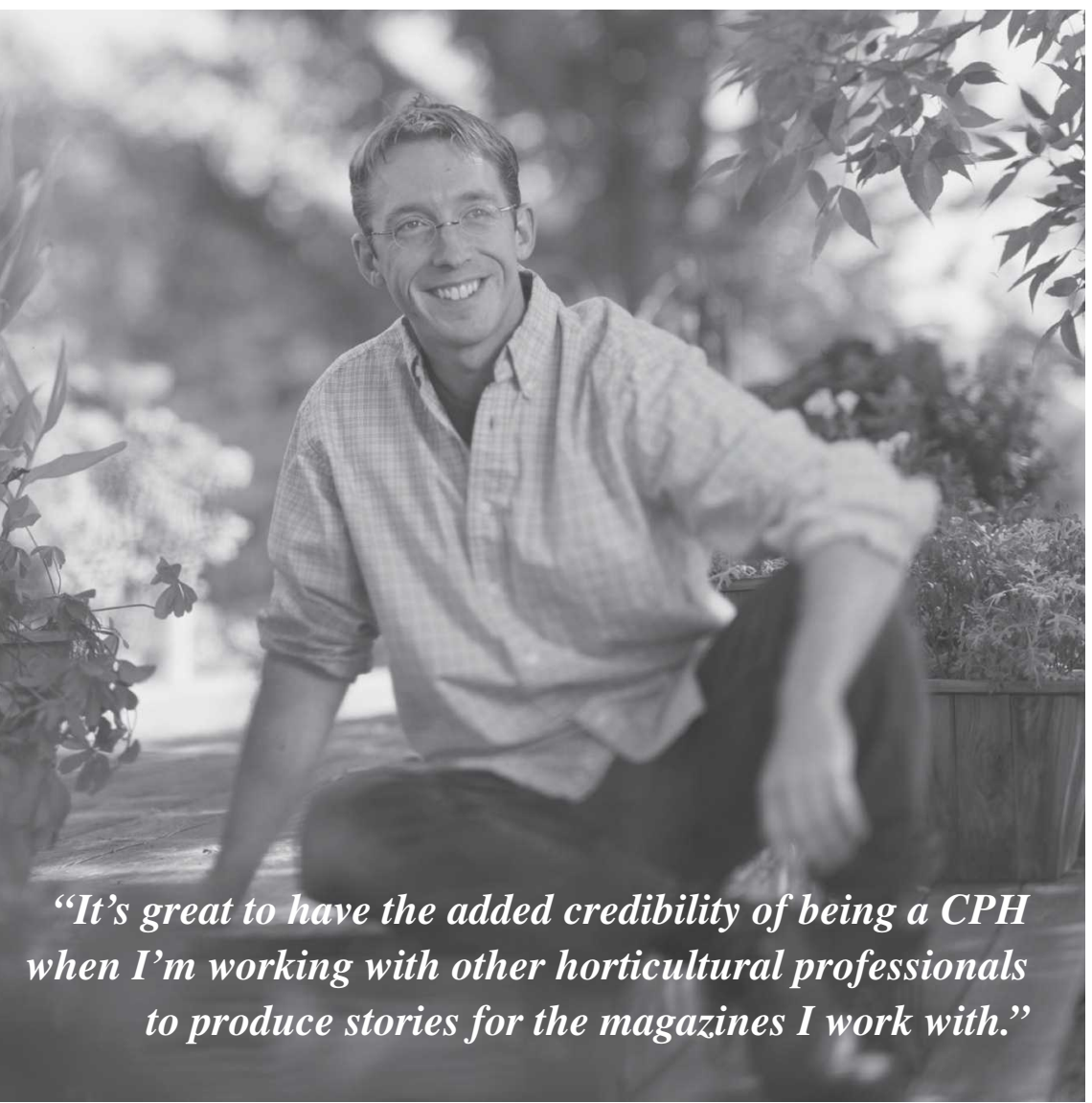

-Justin Hancock, CPH

Editor, Garden Doctor Magazine Better Homes \& Gardens

Special Interest Publications Des Moines, Iowa

\section{${ }^{\star}$ Certified Professional Horticulturist}

The ASHS Certified Professional Horticulturist $(\mathrm{CPH})$ program identifies qualified horticultural professionals for excellence in educational, scientific, and service activities with public and private agencies.

Established in 1991, the CPH program sets standards and maintains procedures for official certification of individuals having recognized credentials in the horticultural field.

A CPH may be sought to:

- consult for industrial and commercial horticulture ventures,

- advise government,

- give legal testimony,

- provide valid information to the media.

In addition to assisting profes- sional advancement, $\mathrm{CPH}$ certification also contributes to the status and visibility of horticulture by:

- promoting and encouraging professional development, growth, and renewal;

- publicizing and exemplifying the CPH Code of Ethics;

- meeting state and national requirements regarding professional recommendations to the public.

For application information and other CPH resources, visit ashs.org on the web. From the home page, select the "Careers" tab at the top of the page. On the Careers page, select "Certified Professional Horticulturist Program." E-mail the CPH program coordinator at: ashscph@ashs.org

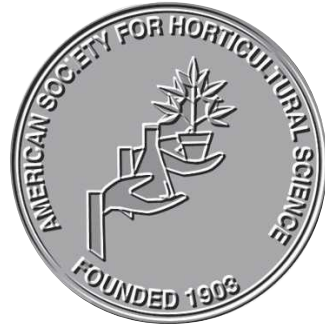

American Society for Horticultural Science

113 S. West St., Suite 200 Alexandria, VA 22314-2851

phone $\cdot 703.836 .4606$

fax $\cdot 703.836 .2024$ web • ashs.org 


\section{Join North America's leading experts on anthracnose management . . .}

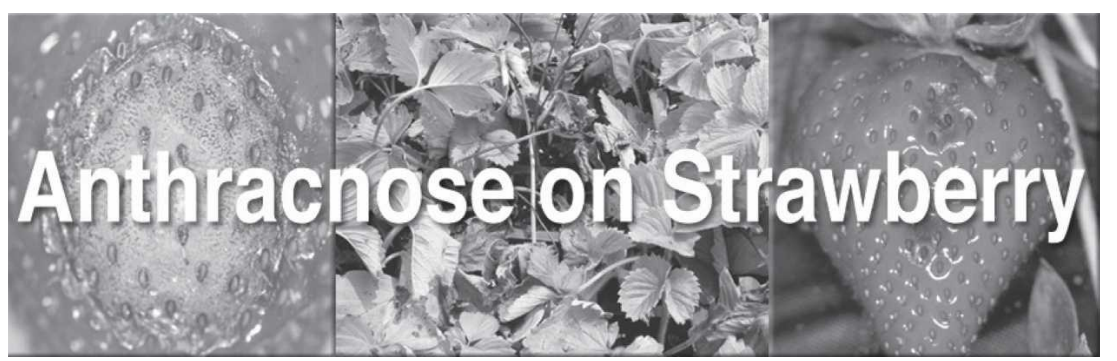

Management Strategies for Plant and Runner Tip Nurseries: A presentation from the ASHS Video Workshop Series that includes more than three hours on three CDs.

\section{... as they discuss the control of Colletotrichum acutatum on strawberry. Nine industry, government, and research experts on strawberry diseases give an in-depth examination of this widespread and difficult-to-manage fruit rot.}

$\Rightarrow$ Includes presentations by: Adam Dale University of Guelph, Ontario, Canada $\bullet$ Stanley Freeman Agricultural Research Organization, The Volcani Center, Bet Dagan, Israel • Curt Gaines University of California, Office of Technology Transfer, Oakland - Kirk Larson University of California, South Coast Research and Education Center, Irvine • Dan Legard California Strawberry Commission, Watsonville • Jim Merteley University of Florida, Gulf Coast Reseach and Education Center, Wimauma • Barclay Poling North Carolina State University, Raleigh • Tom Sjulin Driscoll Strawberry Associates, Watsonville, Calif.

- Zvezdana Pesic-Vanesbroeck North Carolina State University, Micropropagation Center, Raleigh

\section{ORDER ONLINE TODAY:}

http://ashs.org/shop/product.php?productid=16169\&cat=103\&page $=1$ OR: Mail or fax this form with payment to ASHS at the address below.

Name:

Company/University:

Address:

State: Postal Code:

City: Country:

Telephone: Fax:

E-mail:

Payment method: check VISA AMEX

Card \#

exp. date

Signature (req'd.)
ASHS Video Workshop Series

The information on these CDs was presented at the 2004 Annual Conference of the American Society for Horticultural Science in Austin, Texas. This is the first of many CD and DVD products that will be made available from the society.

Title Price Quantity Amount Anthracnose Wkshp. CD set (non-ASHS members) $\$ 49.95$

Anthracnose Wkshp. CD set (ASHS members; co-sponsoring organizations*) $\$ 44.95$

Shipping: US (\$2)

Canada/Mexico (\$5)

International Air (\$10)

VA residents add $5 \%$ sales tax

Total

*North American Strawberry Growers Assn. , California Strawberry Commission, Ontario Berry Growers Assn., Driscoll Strawberry Assocs., Arkansas Strawberry Assn., NC Strawberry Assn. 